\title{
Clinicopathological characteristics of infiltrating lobular breast carcinoma in elderly women: Preliminary results
}

\author{
ALVARO RUIBAL ${ }^{1-4}$, PABLO AGUIAR ${ }^{1-3}$, MARIA DEL CARMEN DEL RÍO ${ }^{5}$, \\ PRIMITIVA MENÉNDEZ ${ }^{6}$, JOSÉ IGNACIO ARIAS ${ }^{7}$ and MICHEL HERRANZ ${ }^{2,3}$
}

\author{
${ }^{1}$ Department of Radiology and Physical Medicine, Faculty of Medicine, University of Santiago de Compostela; \\ ${ }^{2}$ Department of Nuclear Medicine, University Hospital of Santiago de Compostela; ${ }^{3}$ Molecular Imaging Group, IDIS, \\ Santiago de Compostela, 15706 Galicia; ${ }^{4}$ Tejerina Foundation, Madrid 28003; ${ }^{5}$ Clinical Laboratory, Hospital Arquitecto \\ Marcide/Novoa Santos, Ferrol, 15405 Galicia; ${ }^{6}$ Department of Pathology, Central University Hospital of Asturias, \\ Oviedo, 33006 Asturias; ${ }^{7}$ Department of Surgery, Hospital Monte Naranco, Oviedo, 33012 Asturias, Spain
}

Received June 2, 2014; Accepted September 4, 2014

DOI: $10.3892 / \mathrm{mco} .2015 .625$

\begin{abstract}
This study was conducted to investigate the clinicopathological parameters in elderly women (aged $>70$ years) with infiltrating lobular carcinoma (ILC) of the breast and compare the results with those obtained from younger patients (aged 55-70 years). The study sample included a total of 46 women with ILCs, 10 aged $>70$ and 36 aged 55-70 years. The parameters analysed were tumor size, histological grade (HG), axillary lymph node involvement, distant metastasis and immunohistochemical expression of estrogen, progesterone and androgen receptors, Ki67, p53 and B cell lymphoma 2. Compared to women aged 55-70 years, ILCs in women aged $>70$ years were commonly of larger size $(\mathrm{P}=0.068)$ and were more frequently HG3 $(\mathrm{P}=0.024)$. There were no statistically significant differences in the other parameters analysed. Furthermore, we were unable to determine differences in cancer recurrence and mortality in the two patient subgroups during our follow-up. In conclusion, our preliminary results, based on the limited number of cases included in this study, indicate that i) ILCs in women aged $>70$ years tended to be larger compared to those in women aged 55-70 years and were more frequently of grade 3 ; and ii) there were no significant differences in terms of recurrence and mortality between the two patient subgroups during our follow-up.
\end{abstract}

\section{Introduction}

Breast cancer is one of the most common types of cancer in women. Next to gender, the primary risk factor for breast

Correspondence to: Dr Pablo Aguiar, Department of Radiology and Physical Medicine, Faculty of Medicine, University of Santiago de Compostela, Travesía de Choupana s/n, Santiago de Compostela, 15706 Galicia, Spain

E-mail: pablo.aguiar.fernandez@sergas.es

Key words: infiltrating lobular carcinoma of the breast, elderly, $>70$ years, clinicopathological characteristics cancer is age. Over $50 \%$ of breast cancers occur in women aged $\geq 65$ years, $35 \%$ of the patients are aged $>70$ years and $\sim 10 \%$ of breast cancer patients are aged $>80$ years. Similarly, breast cancer is the most common cause of cancer-related mortality in women aged $>65$ years. For this reason, the American Geriatric Society recommends a screening mammography for women aged $\leq 85$ years with a life expectancy of $\geq 3$ years. In addition, the life expectancy of women aged $>70$ years is 15.5 years and half of these women live longer; therefore, their risk may increase significantly. Of note, individuals aged $\geq 65$ years represented $11.3 \%$ of the total population in USA in 1980 and this percentage is expected to increase to $20 \%$ by 2030 (1). Therefore, women aged $>70$ years constitute a group we must focus our attention on due to its marked social significance.

Infiltrating lobular carcinoma (ILC) is the second most common (5-15\%) type of invasive breast cancer following infiltrating ductal carcinoma (IDC). It was previously demonstrated that the incidence rate of lobular carcinoma increased steadily between 1977 and 1995 in women aged $\geq 50$ years, whereas it remained stable in women aged $<50$ years (2). Other investigators consider that the decline in the incidence of ILC may be associated with the reduced use of combined hormone replacement therapy (3). ILC is more likely to present as multifocal, multicentric and bilateral disease; in addition, it exhibits an increasing incidence in patients aged $>50$ years and tends to be sized $>2 \mathrm{~cm}$, have a nuclear grade of $1 / 2$, be estrogen receptor (ER)- and progesterone receptor (PR)-positive, human epithelial grow th receptor 2 (HER2)/neu-negative, exhibit high CD44 expression, low stromal vascular endothelial growth factor receptor 2 expression, no E-cadherin expression and low laminin-5 expression, as compared to IDC (4-7). Other distinguishing characteristics included its association with endocrine responsiveness, patterns of recurrence and overall prognosis (8).

A number of studies have analyzed the metastatic patterns of breast carcinoma and it is currently well known that ILC diverges significantly from IDC in terms of patterns of late local recurrence and sites of distant metastasis $(9,10)$. Borst and Ingold (11) reported that the rates of metastasis to 
the gastrointestinal system (4.5 vs. $0.2 \%)$, reproductive organs (4.5 vs. $0.8 \%$ ), peritoneum-retroperitoneum (3.1 vs. $0.6 \%$ ), adrenal glands ( 0.6 vs. $0 \%)$, bone marrow ( 21.2 vs. $14.4 \%)$ and lung-pleura (2.5 vs. $10.2 \%$ ) were significantly different $(\mathrm{P}<0.05)$ between ILC and IDC, respectively, and were higher in ILC. For this reason, certain investigators recommend including gynecological and gastrointestinal tract evaluation in the follow-up of such patients. Another interesting aspect is that lobular breast cancer patients are more likely to have a father diagnosed with cancer, particularly prostate cancer.

The increased life expectancy of the female population is associated with increased detection of breast tumors in women of advanced age. In this regard, peculiarities in the mammary tumors of women aged $>70$ years, as compared to those of younger patients, were previously reported $(12,13)$. The biological behavior of ILC has not been fully elucidated. Therefore, the aim of this study was to analyze the possible clinicopathological differences in ILC between women aged $>70$ and those aged 55-70 years.

\section{Materials and methods}

Patients. The study group included 46 women with ILC, 10 aged $>70$ and 36 aged 55-70 years, recruited at the Breast Unit of Hospital Monte Naranco (Oviedo, Spain). The parameters analyzed were tumor size, histological grade (HG), axillary lymph node involvement, distant metastasis and immunohistochemical expression of ER, PR, androgen receptor (AR), Ki67, p53 and B cell lymphoma 2 (Bcl-2). The characteristics of the patients are summarized in Table I.

Immunohistochemical analysis. Monoclonal antibodies against ER/PR pharmDx (prediluted clones 1D5 and ER-2123 for ER and PR) 1294 for PR, p53 (DO-7; dilution 1:100; Dako, Carpinteria, CA, USA), Ki67 (MIB-1; dilution 1:200; Dako), Bcl-2 (dilution 1:124; BioGenex, Fremont, CA, USA) and AR (AR441; dilution 1:75; Dako) were used in this study. The ER and PR were assessed according to the Allred score (14) as negative (scores 0-2) and positive (scores 3-8) and the threshold of positivity for p53 and Ki67 was 20 and 15\%, respectively. AR was classified as positive or negative without scoring and Bcl-2 was classified as negative (-), weakly positive (+) and strongly positive (++). A total of 36 women were followed up

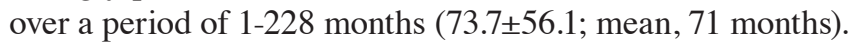

Statistical analysis. SPSS software for Windows (version 20.0; SPSS, Inc., Chicago, IL, USA) was employed for statistical analysis. Continuous variables with a normal Gaussian distribution were expressed as means \pm standard deviation, while non-parametric variables were expressed as median and range. We used the Chi-square test with Yates correction, if necessary, for the comparison of qualitative variables and the Mann-Whitney $\mathrm{U}$ test for continuous variables. $\mathrm{P}<0.05$ was considered to indicate a statistically significant difference.

\section{Results}

Association of patient age with clinicopathological parameters. As shown in Table I, ILC patients aged $>70$ years exhibited larger tumors, compared to those aged 55-70 years,
Table I. Distribution of different clinicopathological parameters in patients with infiltrating lobular carcinoma classified according to age.

\begin{tabular}{lccc}
\hline \multicolumn{3}{c}{ Age, years } \\
\cline { 2 - 3 } Parameters & $\begin{array}{c}>70, \\
\text { no./total } \\
(\mathrm{n}=10)\end{array}$ & $\begin{array}{c}55-70, \\
\text { no./total } \\
(\mathrm{n}=36)\end{array}$ & P-value \\
\hline Size, $\mathrm{cm}$ & & & 0.068 \\
Means \pm SD & $2,5 \pm 1.1$ & $2.0 \pm 1.3$ & \\
Median & 2.4 & 1.5 & \\
Range & $1-5$ & $0.6-6.5$ & \\
N+ & $3 / 10$ & $11 / 36$ & NS \\
N+ $>3$ & $1 / 10$ & $2 / 36$ & NS \\
M+ & $2 / 10$ & $2 / 36$ & NS \\
HG3 & $3 / 10$ & $1 / 36$ & 0.024 \\
ER+ & $10 / 10$ & $36 / 36$ & NS \\
PR+ & $9 / 10$ & $20 / 36$ & NS \\
AR+ & $10 / 10$ & $33 / 36$ & NS \\
Bcl-2+ & $10 / 10$ & $35 / 36$ & NS \\
p53+ & $2 / 10$ & $3 / 36$ & NS \\
Ki67+ & $3 / 10$ & $14 / 36$ & NS \\
Recurrences & $1 / 9$ & $2 / 27$ & NS \\
Metastases & $1 / 9$ & $0 / 27$ & NS \\
\hline
\end{tabular}

$\mathrm{N}$, axillary lymph node involvement; NS, non-significant; $\mathrm{M}$, distant metastasis; HG, histological grade; ER, estrogen receptor; PR, progesterone receptor; AR, androgen receptor; Bcl-2, B-cell lymphoma 2.

with a borderline level of statistical significance $(\mathrm{P}=0.068)$. In addition, the tumors were HG3 in patients aged $>70$ years more frequently compared to those in younger patients; this difference was statistically significant $(\mathrm{P}=0.024)$. The other parameters analyzed, including axillary nodal status, distant metastasis, ER, PR and AR status, Bcl-2, p53 and Ki67, were not statistically significantly different between the two age groups. Furthermore, we were unable to identify differences in the incidence of disease recurrence and mortality between our patient subgroups during the follow-up time.

\section{Discussion}

Breast cancer becomes more prevalent with increasing age; its incidence may increase up to 80 years and plateaus between 80 and 85 years of age (15). IDC remains the most common histological subtype of breast cancer diagnosed in older patients and this type of cancer exhibits an overall more favorable biological profile, with a higher percentage of ER-positive tumors that increases with age to $91 \%$ in patients aged $\geq 85$ years (16). The tumors of older patients are reportedly more often detected by clinical examination rather than imaging techniques (17). Of note, a more elderly cohort (age, $\geq 85$ years) are more likely to present with metastatic 
disease $(9 \%)$ or an unknown stage at the time of diagnosis $(15,17)$. Certain authors consider younger patients to be associated with a worse prognosis compared to older patients; however, the reported findings have been inconclusive.

Schonberg et al (18) reported that breast cancer characteristics appear to be similar between patients aged $\geq 80$ years and younger patients; however, patients aged $\geq 80$ years receive less aggressive treatment compared to their younger counterparts and are more likely to succumb to breast cancer. Fisher et al (19) suggested that, as the life expectancy of women aged $>70$ years may be high, treatment should be based on the histological prognostic characteristics of the primary tumor rather than age alone.

As regards ILC, its incidence was not significantly different between women aged $>70$ and those aged 55-70 years (8.5 and $8.3 \%$, respectively), which was consistent with the findings of Schonberg et al (18); however, the incidence of IDC was lower in women aged $>70$ years compared to that in younger women (70.9 vs. $81.1 \%$, respectively). Fisher et al (19) reported that lobular carcinoma exhibited a gradual increase in incidence with increasing age, with the highest incidence observed among patients aged $>70$ years. ILCs exhibit certain peculiarities compared to IDCs, particularly regarding metastatic behavior, as previously mentioned. However, the behavior of these breast tumors in elderly women (aged $>70$ years) has not been clearly determined, partly due to their lower incidence. Therefore, we aimed to investigate certain clinicopathological parameters and compare the results with those obtained from younger women (aged 55-70 years) with the same type of tumor. We observed that the ILCs of patients aged $>70$ years tended to be of larger size, with a borderline statistically significant difference, whereas they were more frequently HG3, with a statistically significant difference. There were no significant differences in the remaining analyzed parameters or in the incidence of recurrence and mortality between the two age subgroups.

As regards tumor size, our results were consistent with those reported by other groups, indicating that tumor size increases with age and the increase is more significant after the age of 80 years (18). We observed that the characteristics and the clinical and biological behavior of breast carcinomas in women aged $>70$ years differs according to their origin. Tumors tended to be larger, with more frequent axillary node involvement and distant metastases when diagnosed by physical examination during a routine visit; however, for tumors diagnosed through screening, there were no significant differences between women aged $>70$ and those aged 55-70 years (20).

Histological grade is a well known, strong predictor of outcome in patients with invasive breast cancer (21), in whole series as well as in different subgroups based on tumor size and lymph node stage, and it should be incorporated in breast cancer staging systems. Likewise, breast tumor as an entity in elderly women is associated with larger size, a higher number of metastatic lymph nodes, advanced clinical stage, higher HER2 positivity rate, negative ER and PR; it is also associated with triple-negative and HER2 subtypes $(15,22)$. Cuan Martínez et al (12) reported more highly differentiated ductal carcinomas in the group of patients aged $\geq 70$ years. Of note, Fisher et al (19) reported a negative association between high-grade tumors and lymphoplasmacytic reaction; however, the clinical value of the latter has not been clearly established, although it may be associated with a more favorable outcome only in HG3 cases (23).

Our preliminary results, based on the limited number of cases included in the study, indicated that i) ILCs in women aged $>70$ years tend to be larger compared to those in women aged 55-70 years $(\mathrm{P}=0.068)$ and were more frequently HG3 $(\mathrm{P}=0.024)$; and ii) there were no significant differences in terms of recurrence and mortality between the two patient subgroups during our follow-up.

\section{References}

1. Walter LC and Covinsky KE: Cancer screening in elderly patients: a framework for individualized decision making. JAMA 285: 2750-2276, 2001

2. Li CI, Anderson BO, Porter P, et al: Changing incidence rate of invasive lobular breast carcinoma among older women. Cancer 88: 2561-2569, 2000.

3. Eheman CR, Shaw KM, Ryerson AB, Miller JW, Ajani UA and White MC: The changing incidence of in situ and invasive ductal and lobular breast carcinomas: United States, 1999-2004. Cancer Epidemiol Biomarkers Prev 18: 1763-1769, 2009.

4. Fortunato L, Mascaro A, Poccia I, et al: Lobular breast cancer: same survival and local control compared with ductal cancer, but should both be treated the same way? Analysis of an institutional database over a 10-year period. Ann Surg Oncol 19: 1107-1114, 2012.

5. Intra M, Rotmensz N, Viale G, et al: Clinicopathologic characteristics of 143 patients with synchronous bilateral invasive breast carcinomas treated in a single institution. Cancer 101: 905-912, 2004

6. Lesser ML, Rosen PP and Kinne DW: Multicentricity and bilaterality in invasive breast carcinoma. Surgery 91: 234-240, 1982.

7. Gonzalez-Angulo AM, Sahin A, Krishnamurthy S, Yang Y, Kau SW, Hortobagyi GN and Cristofanilli M: Biologic markers in axillary node-negative breast cancer: differential expression in invasive ductal carcinoma versus invasive lobular carcinoma. Clin Breast Cancer 7: 396-400, 2006.

8. Pestalozzi BC, Zahrieh D, Mallon E, et al; International Breast Cancer Study Group: Distint clinical and prognostic features of infiltrating lobular carcinoma of the breast: combined results of 15 International Breast Cancer Study Group clinical trials. J Clin Oncol 26: 3006-3014, 2008.

9. Ferlicot S, Vincent-Salomon A, Medioni J, et al: Wide metastatic spreading in infiltrating lobular carcinoma of the breast. Eur J Cancer 40: 336-341, 2004.

10. Harris M, Howell A, Chrissohou M, Swindell RI, Hudson M and Sellwood RA: A comparison of the metastatic pattern of infiltrating lobular carcinoma and infiltrating duct carcinoma of the breast. Br J Cancer 50: 23-30, 1984.

11. Borst MJ and Ingold JA: Metastatic patterns of invasive lobular versus invasive ductal carcinoma of the breast. Surgery 114: 637-642, 1993.

12. Cuan Martínez JR, Mainero Ratchelous FE, Aguilar Gallegos IU, et al: Comparative study of clinical and pathological features of breast cancer in women with 40 years old and younger vs 70 years old and older. Ginecol Obstet Mex 76: 299-306, 2008 (In Spanish).

13. Ruibal A, Herranz M and Arias JI: Clinical and biological significance of cathepsin D levels in breast cancer cytosol in women over 70 years. Biomark Cancer 4: 1-6, 2012.

14. Allred DC, Harvey JM, Berardo M and Clark GM: Prognostic and predictive factors in breast cancer by immunohistochemical analysis.Modern Pathology 11: 155-168, 1998.

15. Balducci L and Phillips DM: Breast cancer in older women. Am Fam Physician 58: 1163-1172, 1998.

16. Holmes CE and Muss HB: Diagnosis and treatment of breast cancer in the elderly. CA Cancer J Clin 53: 227-244, 2003.

17. Vetter M, Huang DJ, Bosshard G and Güth U: Breast cancer in women 80 years of age and older: a comprehensive analysis of an underreported entity. Acta Oncol 52: 57-65, 2013.

18. Schonberg MA, Marcantonio ER, Li D, Silliman RA, Ngo L and McCarthy EP: Breast cancer among the oldest old: tumor characteristics, treatment choices, and survival. J Clin Oncol 28: 2038-2045, 2010 
19. Fisher CJ, Egan MK, Smith P, Wicks K, Millis RR and Fentiman IS: Histopathology of breast cancer in relation to age. Br J Cancer 75: 593-596, 1997.

20. Ruibal A, Arias JI and Aldecoa B: Clinical and biological differences between infiltrating ductal carcinomas of the breast in women over 70 years-old and those aged 60-70. Med Clin (Barc) 119: 761-764, 2002 (In Spanish).

21. Rakha EA, El-Sayed ME, Lee AH, et al: Prognostic significance of Nottingham histologic grade in invasive breast carcinoma. J Clin Oncol 26: 3153-3158, 2008.
22. Chen ST, Lai HW, Tseng HS, Chen LS, Kuo SJ and Chen DR: Correlation of histologic grade with other clinicopathological parameters, intrinsic subtype, and patients' clinical outcome in Taiwanese women. Jpn J Clin Oncol 41: 1327-1335, 2011.

23. Elston CW, Gresham GA, Rao GS, Zerbo T, Haybittle SL, Houghton J and Kearney G: The cancer resarch campaing (King's/Cambridge trial) for early breast cancer: clinico-pathological aspects. Br J Cancer 45: 655-669, 1982. 